\title{
AVALIAÇÃO DA RETRATIBILIDADE DA MADEIRA DE SETE ESPÉCIES DE Eucalyptus ${ }^{1}$
}

\author{
José Tarcísio da Silva Oliveira², Mario Tomazello Filho³ e Nilton César Fiedler²
}

\begin{abstract}
RESUMO - As variações na retratibilidade no lenho das árvores são as principais causas dos defeitos de secagem, como o empenamento e fendilhamento das peças de madeira. Os tipos de madeira presentes em um tronco estão relacionados com as variações dessa importante propriedade física. Este trabalho teve como objetivo estudar a retratibilidade da madeira de sete espécies de eucaliptos. Os parâmetros de retratibilidade, bem como a sua variação na direção radial da medula em direção à periferia do tronco de sete espécies de eucaliptos, foram avaliados de acordo com a Norma brasileira. De modo geral, os resultados indicaram que as espécies de eucaliptos estudados possuíam madeira com elevada retratibilidade. À exceção das madeiras de Eucalyptus tereticornis e E. pilularis, as demais espécies estudadas apresentaram valores menores de retratibilidade na região próxima da medula.
\end{abstract}

Palavras-chave: Revolvimento de solo, Erosão hídrica e Eucalipto.

EVALUATION OF THE RETRATIBILITY IN SEVEN EUCALYPT SPECIES

\begin{abstract}
The variation of the retratibility in lumber is the main cause of the occurrence of drying defects, especially those characterized by warped and split wood. The different wood types present in the stem are related to the variation of these important physical properties. This work aimed to study the retratibily of seven eucalypt species. The retratibility parameters such as the variation in the pith to bark direction in the peripheral of the trunk were studied according the Brazilian Standard for the seven eucalypts. In general the results showed that the species of eucalypts studied have a high retratibility. Except for Eucalyptus tereticornis and E. pilularis the others species studied showed lower values of retratibility in the pith region.
\end{abstract}

Keywords: Eucalypt wood, Retratibility and Radial variation.

\section{INTRODUÇÃO}

A retratibilidade é, segundo o IPT (1985), o fenômeno da variação dimensional, da madeira, quando há alteração no seu teor de umidade. As variações nas dimensões das peças de madeira começam a ocorrer, quando esta perde ou ganha umidade, abaixo do ponto de saturação das fibras (PSF). O princípio da retratibilidade se deve ao fato de as moléculas de água estarem ligadas por pontes de hidrogênio às microfibrilas dos polissacarídeos que formam a madeira, e quando estas são forçadas a saír, deixam um espaço, e as forças de coesão tendem a reaproximar as microfibrilas, causando, portanto, contração da madeira como um todo. O fenômeno da expansão é o inverso, ou seja, quando a água adsorvida pela madeira, tende a penetrar entre as microfibrilas, causando, portanto, o afastamento delas e o consequente inchamento da peça de madeira como um todo. Basset (1994) enfatizou que essas ligações por pontes de hidrogênio são tão fracas que a água é facilmente liberada, quando a umidade atmosférica cai, permitindo a madeira a se contrair novamente. Uma vez que as madeiras mais densas, por terem maior concentração de células de paredes mais espessas, tendem a absorver mais água por unidade de volume e, consequentemente, a expandir ou contrair mais do que aquelas de menor densidade. Em madeiras densas, mas com elevados teores de extrativos hidrófobos nas paredes celulares, entretanto, as contrações volumétricas poderão não ser muito elevadas.

\footnotetext{
${ }^{1}$ Recebido em 12.10.2007 e aceito para publicação em 02.03.2010.

${ }^{2}$ Universidade Federal do Espírito Santo, UFES, Brasil. E-mail: <jtsilva@npd.ufes.br>.

${ }^{3}$ Universidade de São Paulo, USP, Brasil.
} 
Por ser material anisotrópico, no caso da madeira, mais importante do que avaliar a retratibilidade volumétrica total é ter o conhecimento das variações lineares das dimensões, nas direções transversal e longitudinal. Quanto a esta última, não é preocupante, uma vez que seus valores são muito pequenos, ou menos de 1\% para madeira normal, em todas as espécies. Atenção maior deve ser dada à movimentação transversal nas madeiras, uma vez que estas diferem conforme a direção tangencial ou radial, sendo a primeira maior que a segunda. Kollmann e Cotê (1968) afirmaram que essa diferença entre a retratibilidade radial e a tangencial, que era explicada exclusivamente pela influência restritiva dos raios na direção radial, é também adicionalmente explicada, pelo menos para madeira de coníferas, pelo arranjo helicoidal diferente das microfibrilas nas paredes tangenciais e radiais. Nas paredes celulares radiais dos traqueídeos existem de 50 a 300 pontoações, razão pela qual as microfibrilas são desviadas de seu curso normal. Sem dúvida, uma das causas das propriedades anisotrópicas da madeira é a orientação das micelas, fibrilas e fibras que formam o tecido lenhoso.

Um índice importante, que se obteve a partir dos valores das contrações no sentido transversal, é a relação entre a contração tangencial e a radial, a qual dá uma ideia do comportamento das madeiras, em relação à secagem, indicando maior ou menor propensão de as peças fendilharem. Tais valores variam de 1,3 a 1,4 para madeiras muito estáveis a mais de 3 para espécies extremamente instáveis dimensionalmente, como no caso das madeiras de muitas espécies do gênero Eucalyptus.

Chafe (1987), estudando a influência dos constituintes químicos da madeira de eucalipto, na contração, concluiu que esta foi positivamente relacionada aos teores dos polissacarídeos, sendo negativa a influência de teores de lignina e dos extrativos nessa propriedade.

As características de retração da madeira são bastante diferentes de espécie para espécie florestal, dependendo do modo de condução da secagem e do próprio comportamento da madeira, o que frequentemente leva a alterações da forma e à formação de fendas e empenos. De acordo com Oliveira et al. (2005), as variações de umidade e densidade no lenho das árvores são as principais causas dos defeitos de secagem como o empenamento e fendilhamento das peças de madeiras.
A fim de diminuir as retrações da madeira após o desdobro, ela deve ser seca, ao teor de umidade que corresponde ao de sua aplicação e à do ambiente em que será utilizada.

Em se tratando de madeira proveniente de árvores do gênero Eucalyptus, Hillis (1978) afirmou que na Austrália o crescimento rápido está ligado a contrações excessivas, com o aparecimento de defeitos de secagem, como empenamentos e fendilhamentos, que tendem a ser piores em madeira de menor densidade. Silva et al. (2006), estudando a retratibilidade da madeira de Eucalyptus grandis com idades variando de 10 a 25 anos, concluíram que, de modo geral, a madeira apresentou elevada instabilidade dimensional com valores médios de retratibilidade volumétrica de 18,11\% e retratibilidades radial, tangencial e longitudinal de 6,09\%, $10,14 \%$ e $0,45 \%$ respectivamente, além do fator anisotrópico de 1,71. Para árvores adultas de Eucalyptus saligna, Silva e Oliveira (2003) estudaram as propriedades higroscópicas da madeira para diferentes condições de umidade relativa do ar. Segundo esses autores, os dados de retratibilidade foram bastantes elevados, se comparados com os de outras espécies de madeira de mesma faixa de densidade. Apesar dos elevados coeficientes de contração, esses autores ainda mencionaram que, na madeira de E. saligna estudada, o fator anisotrópico ou relação T/R mostrou-se próximo daquele encontrado na grande maioria das madeiras comerciais brasileiras. Ainda em árvores com idade de 16 anos de E. saligna, Oliveira e Silva (2003) estudaram a variação da retratibilidade e densidade básica da madeira. Tanto para as contrações volumétricas quanto para as lineares, esses autores encontraram valores inferiores de contrações na região medular, com acréscimo para as demais posições radial em direção à periferia do tronco. Quanto ao fator anisotrópico, para essa mesma madeira observou-se decréscimo da região medular para a periferia do tronco.

Pela importância da determinação da retratibilidade, este trabalho teve como objetivo avaliar a retratibilidade na madeira de sete espécies de eucalipto, bem como sua variação na direção radial.

\section{MATERIAL E MÉTODOS}

Neste estudo, utilizou-se a madeira de Corymbia citriodora, E. tereticornis, E. paniculata, E. pilularis, E. cloeziana, E. urophylla e E. grandis, com idade aproximada de 16 anos e 5 árvores de cada espécie 
mencionada de uma plantação da Estação Experimental de Anhembi, SP, do Departamento de Ciências Florestais da ESALQ/USP.

Foram amostradas árvores internas de parcelas de área aproximada de 1 ha de cada espécie estudada. As árvores escolhidas ao acaso no interior das parcelas estiveram dentro de um intervalo de confiança de 5\% para cima ou para baixo em relação ao DAP médio.

A amostragem no sentido radial do tronco no nível do DAP (1,30 m) consistiu na retirada de corpos de prova próximo à medula, a 33 e 66\% do raio, e na periferia do fuste. O procedimento do ensaio seguiu a Norma MB-26/40 da ABNT (1940), porém adotando para cada árvore estudada, um número de 16 corpos de prova de dimensões de $2 \times 2 \times 3 \mathrm{~cm}$, sendo a última dimensão no sentido longitudinal da árvore. Esses corpos de prova foram distribuídos em número de quatro por posição, perfazendo, portanto, um total de 20 amostras por espécie e posição.

\section{RESULTADOS E DISCUSSÃO}

Os valores médios de retratibilidade para a madeira de sete espécies de eucalipto são apresentados na Tabela 1.

Apesar de a retratibilidade volumétrica expressar a variação total ocorrida na variação higroscópica, as lineares que ocorreram ao longo dos planos de orientação da madeira são na maioria das vezes mais importantes, devido ao fato de serem diferentes, tornando a madeira material anisotrópico. Principalmente em se tratando da variação dimensional na direção transversal (radial e tangencial), há desequilíbrio entre os valores da retratibilidade. Tal desbalanceamento entre as contrações é chamado de fator anisotrópico, ou relação entre a retratibilidade na direção tangencial dividida pela mesma propriedade na direção radial.

A retratibilidade total na direção radial, apresentada na Tabela 1, foi significativamente mais alta para a madeira de E. paniculata e significativamente mais baixa para as espécies de E. cloeziana e E. grandis, respectivamente. Alguns autores como Kollmann e Cotê (1968) afirmaram que, quanto mais alta a densidade da madeira, maior a sua contração ou expansão, porém, principalmente para o caso do gênero Eucalyptus, há controvérsia em tal afirmação. A Tabela 1, já contradiz esta afirmação, com espécies de elevada densidade, a exemplo do E. cloeziana e C. citriodora, apresentando madeiras com menores valores de retratibilidade do que no caso das madeiras de E. urophylla e E. pilularis. Os valores determinados neste estudo estão condizentes com outros obtidos para madeira adulta, por diversos autores como IPT (1956), Albuquerque (1991), Carmo (1996), Lima (1996), além de informações obtidas e não publicadas pelo próprio autor.

Quanto à variabilidade, apresentada pelos coeficientes de variação da Tabela 1, para a maioria das espécies em estudo, pode ser considerada elevada. A afirmação do Forest Products Laboratory (1987) mostra que, para um estudo baseado em 50 espécies, o

Tabela 1 - Valores médios de retratibilidade (\%) e fator anisotrópico da madeira de sete espécies de Eucalyptus. Table 1 - Average of the retratibility (\%) and anisotropic factor to the seven wood species of Eucalyptus.

\begin{tabular}{|c|c|c|c|c|c|c|}
\hline \multirow[t]{2}{*}{ ESPÉCIE } & \multirow[t]{2}{*}{$\mathrm{N}^{1}$} & \multicolumn{4}{|c|}{ RETRATIBILIDADE (\%) } & \multirow{2}{*}{$\begin{array}{c}\text { Fator } \\
\text { Anisotrópico }\end{array}$} \\
\hline & & Radial $^{3}$ & Tangencial & Axial & Volumétrica & \\
\hline E. $P$ & 80 & $4 \mathrm{~A}(1$, & $5,5 \mathrm{~A}(2$ & $3(0,09)($ & $27,2 \mathrm{~A}$ & $1,7 \mathrm{DE}$ \\
\hline E. urophylla & 0 & $9 \mathrm{~B}(1$, & ,3 A(4 & ,1) & $25,6 \mathrm{~A}$ & $2,0 \mathrm{BC}$ \\
\hline E. tereticornis & 80 & 7,3 ВС $(1,2)(17,0)$ & $13,2 \mathrm{~B}(3,6)(27,5)$ & $3(0,1)(33,3)$ & $22,3 \mathrm{~B}(4,6$ & $1,8 \mathrm{CD}(0$ \\
\hline E. pilularis & 80 & ,4 DE $(2,0)(30,1)$ & $2,9 \quad \mathrm{~B}(3,7)(28,6)$ & $(0,2$ & $21,5 \quad B(4$ & $2,2 \mathrm{~A}($ \\
\hline C. citriodora & 80 & $7,1 \mathrm{CD}(1,5$ & $10,0 \quad C(1,4)(14,1)$ & $0,2(0,1)(48,8)$ & $18,3 \mathrm{C}(2,7)(14,8)$ & $1,4 \mathrm{E}(0$, \\
\hline E. cloeziana & 80 & $5,7 \mathrm{EF}(1,3$ & $3 \mathrm{C}(1,6)(17,1)$ & 0) & $16,5 \mathrm{CD}(2,7)(16,3)$ & $1,7 \mathrm{DE}(0,3)(19,3)$ \\
\hline E. grandis & 80 & $5,0 \quad F(1,4)(33,0)$ & $10,1 \quad C(2,5)(25,0)$ & $0,4(0,1)(26,3)$ & $15,9 \mathrm{D}(4,2)(26,1)$ & $2,1 \mathrm{AB}(0,5)(21,8)$ \\
\hline
\end{tabular}

1 - Número de observações.

2 - Valores entre parêntesis são desvio-padrão e coeficiente de variação em (\%), respectivamente.

3 - Médias seguidas pela mesma letra na vertical não diferem estatisticamente pelo teste de Tukey a 5\% de significância.

1 - Número de observações.

2 - Valores entre parêntesis são desvio-padrão e coeficiente de variação em (\%), respectivamente.

3 - Médias seguidas pela mesma letra na vertical não diferem estatisticamente pelo teste de Tukey a 5\% de significância. 
coeficiente de variação fica próximo de 15\% para as contrações volumétricas e transversais. Para madeira de eucalipto, porém, tais valores são esperados serem mais elevados. A madeira de E. grandis, apesar de possuir o valor mais baixo de retratibilidade na direção radial, apresenta o maior coeficiente de variação, demonstrando aí, mais uma vez como em discussão anterior, a maior heterogeneidade de seu lenho.

No caso da retratibilidade tangencial, mostrada na Tabela 1, o maior valor foi apresentado para a madeira de E. paniculata, sendo, porém, estatisticamente não diferente daquela produzida por E. tereticornis. Valores mais baixos, por volta de $10,0 \%$, são apresentados para a madeira de C. citriodora, E. cloeziana e E. grandis, que são iguais estatisticamente. Observa-se também, neste caso, que a exceção dos coeficientes de variação inferiores, apresentados pelas madeiras de E. paniculata (14,7\%), e C. citriodora (14,1\%), às demais apresentam esses valores bastante elevados. Durlo e Marchiori (1992) afirmaram que os valores da contração tangencial oscilam em torno do dobro daquelas das contrações radiais e são cerca de 20 vezes aos detectados no sentido longitudinal ou axial, isso para a madeira de modo geral. Para espécies de eucaliptos, essa relação entre as contrações transversais poderá ultrapassar três vezes. Acredita-se que na madeira de folhosas as maiores diferenças entre a retratibilidade tangencial em relação à radial são atribuídas às características anatômicas. Principalmente o volume de raio, em que quanto maior o número de células, que constituem sua largura e altura, maior a contribuição em termos da restrição da variação dimensional na direção radial. Entretanto, outros fatores como as dimensões radiais das fibras e diferenciação química entre as paredes celulares radiais e tangenciais também podem, segundo Masseran e Mariaux (1985), ser fatores decisivos a influenciar na anisotropia da retratibilidade das madeiras.

Quanto aos valores de retratibilidade obtidos na direção axial ou longitudinal da madeira de eucalipto mostrados na Tabela 1, optou-se por não submeter tais valores ao teste estatístico, em primeiro lugar devido à baixa variação entre espécies e, segundo, devido aos valores extremamente elevados de coeficientes de variação apresentados. Kollmann e Cotê (1968) afirmaram que a contração longitudinal total de madeira normal varia entre 0,1 e $0,9 \%$ e que para a maioria dos usos uma contração axial total uniforme de $0,3 \%$ está próximo do permissível. Tal valor se aproxima para a maioria das madeiras deste estudo. Esses autores ainda afirmaram que a contração longitudinal varia inversamente com a densidade da madeira. Albuquerque (1991) apresentou valores mais elevados do que os encontrados aqui, também para madeira adulta, e com variação elevada, representada pelos elevados desvios-padrão. Essa autora apresentou os seguintes valores para as madeiras de E. paniculata $(0,9)$, E. urophylla $(1,1)$, E. tereticornis $(0,9)$, C. citriodora $(0,8)$ e E. cloeziana $(0,6 \%)$. Segundo oFOREST PRODUCTS LABORATORY (1987), os valores médios de contração longitudinal total estão entre 0,1 e $0,2 \%$ para a maioria das espécies. Madeira anormal, como de reação, lenho juvenil e de grã-revessa, apresenta valor mais elevado. Sabe-se que o principal componente a influenciar essas contrações é o ângulo microfibrilar que, quanto maior, mais elevados são esses valores.

A retratibilidade volumétrica da madeira apresentada na Tabela 1 difere entre madeiras das espécies de eucalipto. De maneira geral, as espécies em estudo possuem madeira de elevada retratibilidade, característica comum às madeiras do gênero, principalmente aquelas advindas de árvores de rápido crescimento. Importante é observar os valores do desvio-padrão e coeficiente de variação, os quais indicam que, apesar de produzir madeira de elevada retratibilidade, em $E$. paniculata a variabilidade do parâmetro é relativamente baixa. No entanto, $E$. grandis, apesar de produzir madeira de menor retratibilidade, apresentou a mais elevada variabilidade em relação ao parâmetro, o que inevitavelmente está relacionado com a ocorrência de defeitos na fase de secagem e, mesmo, devido a variações bruscas na sua umidade de equilíbrio higroscópico. Apesar de maior densidade, madeira de E. cloeziana não difere estatisticamente daquela de E. grandis, quanto a tal propriedade, entretanto valores nitidamente inferiores de desvio-padrão e coeficiente de correlação mostrados pela primeira espécie, já por si só asseguram qualidade superior dessa madeira, quanto à utilização que pressuponha maior uniformidade do tecido lenhoso, o qual, por sua vez, refletirá em maior estabilidade de desempenho. Raciocínio análogo poderá ser feito em relação às madeiras de $E$. paniculata e E. urophylla, quanto à variabilidade destacadamente inferior da primeira, apesar de apresentarem valores idênticos, estatisticamente, quanto à retratibilidade volumétrica total. 
O fator anisotrópico é a relação entre a retratibilidade na direção tangencial dividida pela mesma propriedade na direção radial. A situação ideal, raramente encontrada, seria aquela na qual as tensões decorrentes da natureza anisotrópica se anulariam segundo as direções em que a retratibilidade se manifestasse. A importância desse índice, portanto, é que, quanto maior for o seu distanciamento da unidade, mais propensa será a madeira a fendilhar e empenar durante as alterações dimensionais provocadas pela variação higroscópica. Apesar de ser comum encontrar valores extremamente elevados, para esse parâmetro, quando se trata de madeira de eucalipto, principalmente aquela proveniente de árvores jovens e de rápido crescimento, os valores apresentados pela Tabela 1 podem ser considerados bastante satisfatórios, de modo geral.

Estatisticamente, madeiras de E. pilularis e E. grandis se destacam de forma negativa daquelas produzidas pelas demais espécies. Destaca-se também o baixo valor desse parâmetro, para a madeira de $C$. citriodora, o que, consequentemente, a caracteriza como portadora de estrutura lenhosa de alta estabilidade dimensional. Tal propriedade já foi comprovada no desempenho altamente satisfatório observado na operação de desdobro das toras. O fator anisotrópico apresentado para a madeira de $C$. citriodora compara com aquele da madeira de mogno (Swietenia macrophylla), considerada uma das madeiras de maior estabilidade dimensional existente no mundo. Tal característica é sem dúvida alguma, em grande parte, responsável por ser o mogno uma das espécies mais valorizadas no mercado internacional.

Madeiras de E. paniculata, E. tereticornis e E. cloeziana são estatisticamente não diferentes em relação a esse parâmetro. Carmo (1996) encontrou para madeira de 21 anos de idade os seguintes valores de fator anisotrópico: E. grandis (1,65), C. citriodora $(1,12)$, E. pilularis $(1,61)$ e E. cloeziana $(1,76)$. Estudos publicados pelo IPT (1956), apontam para E. paniculata (1,93), C. citriodora (1,44), E. tereticornis $(1,94)$ e E. pilularis $(2,15)$.

Durlo e Marchiori (1992) apresentaram o seguinte critério de classificação da madeira quanto a esse parâmetro: 1,2-1,5 - considerado excelente, ocorrendo em madeira de cedro, sucupira, mogno, balsa entre outras; 1,5-2,0 - normal, exemplificados em ipê, pinus, araucária, peroba-rosa e teca, entre outras espécies; e acima de 2,0 - como ruim, que pode ocorrer em madeiras de araucária, imbúia, álamo e jatobá, entre outras espécies. De acordo com as classes mencionadas pelos autores anteriormente citados, madeira de C. citriodora poderá ser considerada excelente; a de E. paniculata, E. tereticornis, E. cloeziana e, ainda, E. urophylla, com comportamento normal; e somente a madeira produzida pelas espécies de E. grandis e E. pilularis poderiam ser consideradas um fracasso quanto à sua estabilidade dimensional. Diversos critérios de classificação das madeiras, quanto aos parâmetros de retratibilidade, são apresentados na literatura, podendo destacar, entre eles, aqueles propostos por Sallenave (1971), que considerou as retratibilidades lineares transversais e a volumétrica e o apresentado por Nahuz (1974), também levando em conta os mesmos parâmetros citados, porém com maior número de faixas de classificação.

Na Tabela 2, observa-se que, quanto a retratibilidade volumétrica total, as madeiras de C. citriodora, $E$. tereticornis, E. urophylla e E. grandis tiveram variações altamente significativas, entre suas árvores. Para a madeira de E. pilularis e E. cloeziana, apesar da

Tabela 2-Sumário da análise de variância da retratibilidade volumétrica e fator anisotrópico totais da madeira de sete espécies de Eucalyptus.

Table 2 - Sumary of variance analysis to volumetric retratibility and anisotropic factor to the seven species wood of Eucalyptus.

\begin{tabular}{lccc}
\hline ESPÉCIE & PROPRIEDADE & \multicolumn{2}{c}{ FONTE DE VARIAÇÃO } \\
\cline { 3 - 4 } & & $\begin{array}{c}\text { Árvore } \\
(4)^{2}\end{array}$ & $\begin{array}{c}\text { Posição } \\
\text { Radial } \\
(3)\end{array}$ \\
\hline C. citriodora & 12 & $* * * *$ & $* * * *$ \\
E. tereticornis & 12 & $* *$ n.s. & $* * * *$ \\
E. paniculata & 12 & n.s. & n.s. $* *$ \\
E. pilularis & 12 & $* * *$ & n.s. $* *$ \\
E. cloeziana & 12 & $* *$ & $* * * *$ \\
E. urophylla & 12 & $* * * *$ & $* *$ \\
E. grandis & 12 & $* *$ n.s. & $* * * *$ \\
\hline
\end{tabular}

1 - A propriedade 1 é a retratibilidade volumétrica e a 2, o fator anisotrópico.

2 - Graus de liberdade.

* - Significativo a 5\% de probabilidade.

** - Significativo a $1 \%$ de probabilidade.

n.s - Não significativo.

1 - A propriedade 1 é a retratibilidade volumétrica e a 2, o fator anisotrópico.

2 - Graus de liberdade.

* - Significativo a 5\% de probabilidade.

**-Significativo a $1 \%$ de probabilidade.

n.s - Não significativo.

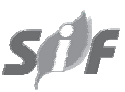

Revista Árvore, Viçosa-MG, v.34, n.5, p.929-936, 2010 
ocorrência de variação entre árvores, ainda em relação à propriedade mencionada anteriormente, esta foi verificada em um menor nível de probabilidade. Na madeira de E. paniculata, não houve diferença significativa, entre árvores, quanto a essa propriedade. Quanto ao fator anisotrópico, este foi altamente variável entre as árvores de C. citriodora, E. pilularis e E. urophylla. Também variável, porém em menor significância entre as árvores de E. paniculata e E. cloeziana. Madeira de E. tereticornis e E. grandis não apresentaram variações significativas entre árvores, com relação a esse índice.
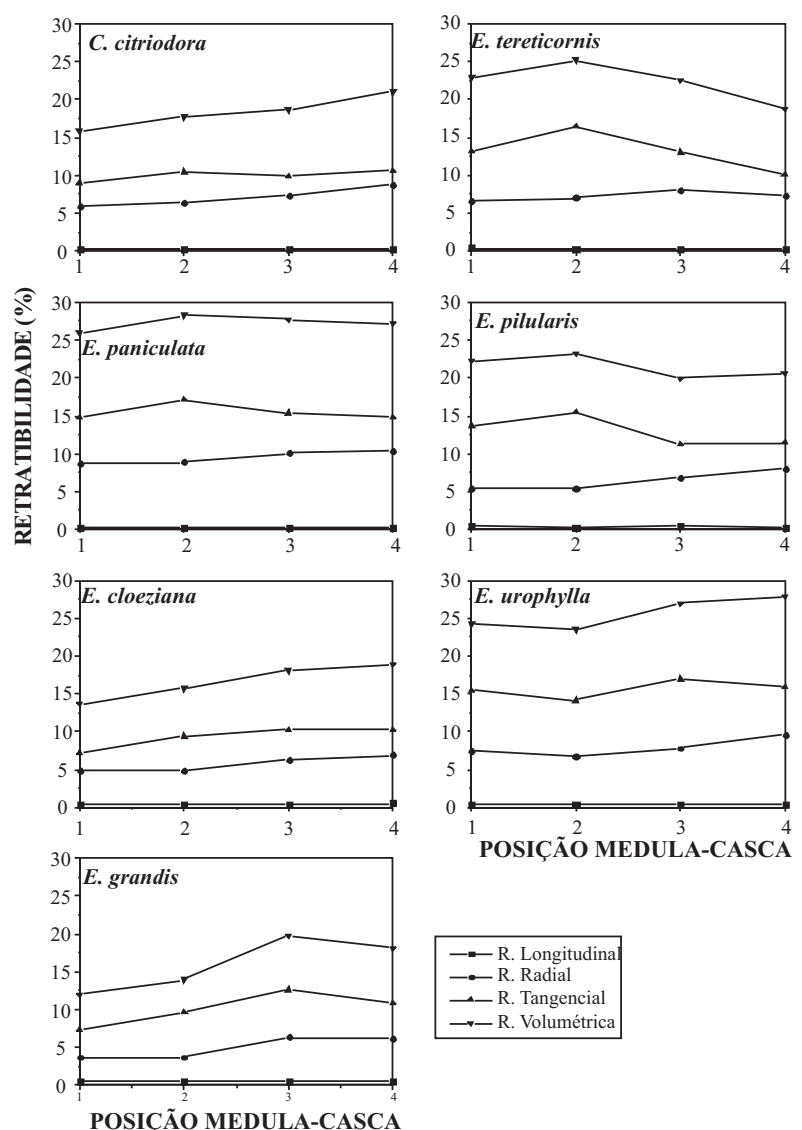

Figura 1 - Representação gráfica da variação das retratibilidades linear e volumétrica totais em \%, em quatro posições eqüidistantes na direção medula-casca no DAP da madeira de sete espécies de Eucalyptus.

Figure 1 - Graphic representation of the variation for the total linear and volmetric retratibility at four equal positions in the pith to bark direction at the diameter at breast height level for seven eucalypt species.
Na posição radial, em quatro posições equidistantes, a partir da medula em direção à periferia do tronco, a análise da Tabela 2, mostra comportamento altamente variável nos dois parâmetros nas madeiras de $C$. citriodora, E. cloeziana e E. grandis. Em E. urophylla, tais variações também ocorreram em ambos os parâmetros, porém em um nível de probabilidade inferior. Madeiras de E. pilularis e E. cloeziana comportaram-se de maneira semelhante, não apresentando variação radial significativa em relação à retratibilidade volumétrica, mas sendo altamente variável em relação ao fator anisotrópico. Essa análise deve ser feita levando em consideração não somente a variação desses parâmetros, mas também sua magnitude, apresentados de forma geral na Tabela 1.

Para a madeira de eucalipto deste estudo, a variação das contrações lineares e volumétricas totais, em quatro posições equidistantes no DAP, é apresentada de forma gráfica na Figura 1.

A Figura 1 deixa claro o padrão crescente de retratibilidade, na direção medula-casca, para a madeira das espécies de C. citriodora, E. cloeziana e E. urophylla. Para todas as madeiras, a exceção daquela de E. tereticornis e E. pilularis, apresentaram valores menores de retratibilidade na região medular. A distribuição de umidade no interior das árvores dessas sete espécies de eucalipto deste estudo foi estudada por Oliveira et al. (2005), que relataram maior homogeneidade de distribuição de umidade dentro das árvores de E. paniculata e C. citriodora. Esses mesmos autores relataram que o diferencial de umidade da madeira nas regiões internas do tronco de E. paniculata e $C$. citriodora foi de $20 \%$ e de E. urophylla e E. grandis de $80 \%$. Observação importante, que deve ser considerada nos gráficos da Figura 1, é a diferença entre as contrações radial e tangencial, apresentada para as diferentes madeiras. Para a madeira de $C$. citriodora e E. cloeziana, essa diferença é relativamente pequena, principalmente se comparada com as madeiras de E. tereticornis, E. pilularis e também E. urophylla. Observa-se também que essa diferença tende a diminuir, da posição 3 para a 4 (periferia), na madeira das espécies de C. citriodora, E. paniculata, E. cloeziana, E. tereticornis e E. pilularis. Em E. urophylla e E. grandis, tal queda não é claramente verificada. A posição 4, para todas as madeiras, é predominantemente constituída de alburno. 
Carmo (1996) encontrou na madeira de E. grandis de 21 anos de idade tendência de aumento da contração volumétrica no sentido da casca, com redução nas camadas mais periféricas, comportamento esse, portanto, similar ao apresentado no gráfico da Figura 1. Com relação às madeiras de C. citriodora e E. cloeziana, essa mesma autora afirmou ser a variabilidade quase nula, porém com tendência a aumentar na direção radial, apesar de homogêneas.

\section{CONCLUSÕES}

Com base nos resultados apresentados e discutidos com relação à madeira de sete espécies de Eucalyptus e relativos à retratibilidade, conclui-se que:

- De modo geral, as espécies em estudo possuem madeira de elevada retratibilidade, estando, ainda assim, dentro dos limites apresentados na literatura.

- Os valores de fator anisotrópico encontrados na madeira deste estudo, de maneira geral, podem ser considerados bastante satisfatórios. Devendo a madeira de $C$. citriodora ser considerada excelente e a de $E$. paniculata, E. tereticornis, E. cloeziana e ainda E. urophylla de comportamento normal, e somente a madeira produzida por E. grandis e E. pilularis, pode ser considerada um fracasso quanto à sua estabilidade dimensional.

- À exceção das madeiras de E. tereticornis e E. pilularis, as demais apresentaram valores menores de retratibilidade na região próxima da medula. Não houve, portanto, padrão definido para a variação da retratibilidade na direção medula-casca da madeira deste estudo.

- Torna-se errônea a generalização, mesmo em se tratando de um único gênero, como é o caso dos eucaliptos, com respeito às relações entre retratibilidade e densidade, uma vez que, dentro de um número limitado de espécies deste estudo, o comportamento das madeiras foi altamente variável.

\section{REFERÊNCIAS}

ALBUQUERQUE, M. C. J. Indicação para o emprego de dezesseis espécies de eucalipto na construção civil. 1991. 134p. Dissertação (Mestrado) - Escola de Engenharia de São Carlos, São Carlos, 1991.
ASSOCIAÇÃO BRASILEIRA DE NORMAS TÉCNICAS. - ABNT. Ensaios físicos e mecânicos da madeira - MB-26/40. Rio de Janeiro: 1940. 16p.

BASSET, G. Swelling, shrinking and fire resistence. Asian Timber, v.13, n.10, p.28-33, 1994.

CARMO, A. P. T. Avaliação de algumas propriedades da madeira de seis espécies de eucalipto. 1996.74f. Dissertação(Mestrado) - Universidade Federal de Viçosa, Viçosa, MG, 1996.

CHAFE, S. C. Collapse, volumetric shrinkage, specific gravity and extratives in Eucalyptus and other species. Wood Science and

Technology, v.21, n.1, p.27-41, 1987.

DURLO, M. A.; MARCHIORI, J. N. C. Tecnologia da madeira: retratibilidade. Santa Maria: CEPEF/FATEC, 1992. 33p. (Série Técnica, 10)

\section{FOREST PRODUCTS LABORATORY. Wood}

handbook: wood as an engineering material. Washington: U.S. Department of Agriculture, 1987. 466p. (Agriculture Handbook, 72)

ILLIS, W. E.; BROWN, A. G. (Eds.)

Eucalipts for wood production. Melbourn: CSIRO, 1978.

INSTITUTO DE PESQUISAS TECNOLÓGICAS IPT. Madeira: o que é e como pode ser processada e utilizada. São Paulo: 1985. 189p. (Boletim ABPM, 36)

\section{INSTITUTO DE PESQUISAS TECNOLÓGICAS - \\ IPT. Métodos de ensaios adotados no IPT para o estudo de madeiras nacionais, Tabelas de resultados obtidos para madeiras nacionais, nomenclatura das madeiras nacionais. 2.ed. São Paulo: IPT, 1956. 62p. (Boletim Técnico, 31)}

KOLlmAnN, F. F. P.; COTÊ, W. A. Principles of wood science and technology. New York: Springer-Verlag, 1968. v.1.

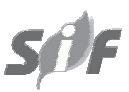

Revista Árvore, Viçosa-MG, v.34, n.5, p.929-936, 2010 
LIMA, T.G. Variações no sentido radial e longitudinal de algumas propriedades das madeiras de Eucalyptus microcorys F. Muell e Eucalyptus pilularis Sm. 1996.

106p. Dissertação (Mestrado) - Universidade Federal de Viçosa, Viçosa, MG, 1996.

MASSERANN, C.; MARIAUX, A. Anisotropie de retrait et structure du bois. Recherche de l'influence des caractéres morphologiques transverses des fibres. Bois et Forêts des Tropiques, n.209, p.35-47, 1985.

NAHUZ, M. A. R. Some aspects of the introduction of lesser known brazilian species to the european timber market. 1974. 243f. Thesis (Magister in Scientiae) University College of North Wales, Bangor, 1974.

OLIVEIRA, J. T. S. et al. Variação do teor de umidade e da densidade básica na madeira de sete espécies de eucalipto. Revista Árvore, v.29, n.1, p.115-127, 2005.
OLIVEIRA, J. T. S.; SILVA, J. C. Variação radial da retratibilidade e densidade básica da madeira de Eucalyptus saligna Sm. Revista Árvore, v.27, n.3, 381-385, 2003.

SILVA, J. C.; OLIVEIRA, J. T. S. Avaliação das propriedades higroscópicas da madeira de Eucalyptus saligna Sm., em diferentes condições de umidade relativa do ar. Revista Árvore, v.27, n.2, p.233-239, 2003.

SILVA, J. C. et al. Variação da retratibilidade da madeira de Eucalyptus grandis Hill ex. Maiden, em função da idade e da posição radial no tronco. Revista Árvore, v.30, n.5, p.803-810, 2006.

SALlenaVe, P. Proprietés physiques et mécaniques des bois tropicaux. Paris: Centre Technique Forestier Tropical. France, deuxieme supplement, 1971. 123p. 